\title{
Numerical Solution of a Boundary Value Problem Including Both Delay and Boundary Layer
}

\section{Erkan Cimen}

\author{
Van Yuzuncu Yil University \\ 65080, Van, Turkey \\ E-mail(corresp.): cimenerkan@hotmail.com
}

Received December 19, 2017; revised September 14, 2018; accepted September 5, 2018

\begin{abstract}
Difference method on a piecewise uniform mesh of Shishkin type, for a singularly perturbed boundary-value problem for a nonlinear second order delay differential equation is analyzed. Also, the method is proved that it gives essentially first order parameter-uniform convergence in the discrete maximum norm. Furthermore, numerical results are presented in support of the theory.
\end{abstract}

Keywords: singular perturbation, boundary-value problem, finite difference method, delay differential equation, Shishkin mesh.

AMS Subject Classification: 34K10; 34K26; 65L11; 65L12; 65L20.

\section{Introduction}

If an ordinary differential equation includes not only a perturbation parameter but also a delay term, it is called a singularly perturbed differential-difference equation. These equations arise frequently in science and engineering field. For instance, they occur in the study of human pupil light reflex [19], first-exit problems in neurobiology [24], models of physiological processes and diseases [20], optimal control theory [13], models of climate systems [15], optically bistable devices [7] and signal transmission [9], and other models [10].

On the other hand, in recent years many methods have been developed for solving singularly perturbed delay differential problems. In $[4,16,17]$ have been considered some asymptotic analysis of boundary value problems for second order singularly perturbed differential-difference equations and some numerical techniques for solving of this type of problems with large and small shifts were considered in $[12,14,18]$ and references therein. Particularly, reproducing 
kernel method [12], initial value technique [25], some special finite element method $[23,26]$ have been used for solving these problems.

Motivated by the above works, we are interested in the following model problem:

$$
L u:=\varepsilon u^{\prime \prime}(x)+a(x) u^{\prime}(x)+f(x, u(x), u(x-r))=0, x \in \Omega,
$$

subject to the interval and boundary conditions,

$$
u(x)=\varphi(x), x \in \Omega_{0} ; u(l)=A,
$$

where $\Omega=\Omega_{1} \cup \Omega_{2}, \Omega_{1}=(0, r], \Omega_{2}=(r, l), \bar{\Omega}=[0, l], \Omega_{0}=[-r, 0]$ and $0<\varepsilon \ll 1$ is the perturbation parameter, $a(x) \geq \alpha>0, f(x, u, v)$, and $\varphi(x)$ are given sufficiently smooth functions satisfying certain regularity conditions in $\bar{\Omega}, \bar{\Omega} \times \mathbb{R}^{2}$ and $\Omega_{0}$ respectively, to be specified and $r$ is a constant delay, which is independent of $\varepsilon$, and $A$ is a given constant and furthermore

$$
0 \leq \frac{\partial f}{\partial u} \leq b^{*}<\infty, \quad\left|\frac{\partial f}{\partial v}\right| \leq c^{*} .
$$

The special case $r=0$ and $\varphi(x)=B$ ( $B$ a real constant) of the above problem so called two-point boundary value problem of singularly perturbed differential equations and typically boundary layers occur in their solutions. Such problems appear in the Navier-Stokes equations of fluid flow at high Reynolds number, the equations governing flow in a porous medium, the drift-diffusion equations of semiconductor device physics, fluid mechanics, elasticity, quantum mechanics, plasticity, oceanography, meteorology, reaction-diffusion processes and mathematical models of liquid materials and of chemical reactions [21,22].

Besides, for small values of $\varepsilon$, standard numerical methods for solving such problems are unstable and do not give accurate results. Therefore, it is important to develop suitable numerical methods for solving these problems, whose accuracy does not depend on the parameter value $\varepsilon$, i.e., methods that are convergent $\varepsilon$-uniformly. These include fitted finite difference methods, finite element methods using special elements such as exponential elements, and methods which use a priori refined or special non-uniform grids which condense in the boundary layers in a special manner. One of the simplest ways of derive parameter-uniform methods consists of using a class of special piecewise uniform meshes, such as Shishkin meshes (see [6,11,21, 22] for the motivation for this type of mesh), which are constructed a priori and depend on the parameter $\varepsilon$, the problem data, and the number of corresponding mesh points. The various approaches to the design and analysis of appropriate numerical methods for singularly perturbed differential equations can be found in $[8,11,21,22]$ (see also references cited in them). The numerical method presented here comprises a finite-difference scheme on a Shishkin type mesh. We have derived this approach on the basis of the method of integral identities with the use of interpolating quadrature rules with the weight and remainder terms in integral form. These results in a local truncation error containing only first second derivatives of exact solution and hence facilitates examination of 
the convergence. The solution of a singularly perturbed problem of the form (1.1)-(1.2) normally has a boundary layer at $x=0$.

The remainder paper is organized as follows. In Section 2, we state some important properties of the exact solution. The finite difference discretization is introduced in Section 3. In Section 4, we present the error analysis for the approximate solution. Uniform convergence is proved in the discrete maximum norm. In Section 5, we formulate the iterative algorithm for solving the discrete problem and present numerical results which validate the theoretical analysis computationally. The paper ends with a summary of the main conclusions.

Henceforth, $C$ denotes a generic positive constant independent of $\varepsilon$ and the mesh parameter. Some specific, fixed constants of this kind are indicated by subscripting $C$. For any continuous function $g(x)$ denote norms which

$$
\begin{aligned}
& \|g\|_{\infty} \equiv\|g\|_{\infty, \bar{\Omega}}=\max _{0 \leqslant x \leqslant l}|g(x)|, \quad\|g\|_{1} \equiv\|g\|_{1, \Omega}=\int_{0}^{l}|g(x)| d x \\
& \|g\|_{\infty, k} \equiv\|g\|_{\infty, \bar{\Omega}_{k}}, \quad\|g\|_{1, k} \equiv\|g\|_{1, \Omega_{k}}, \quad k=0,1,2 .
\end{aligned}
$$

\section{Properties of exact solution}

Firstly, we show some properties of the solution of (1.1)-(1.2), which are needed in later sections for the analysis of appropriate numerical solution.

Lemma 1. Let $a(x) \in C(\bar{\Omega}), f(x, \cdot, \cdot) \in C^{1}\left(\bar{\Omega}, \mathbb{R}^{2}\right), \varphi(x) \in C\left(\Omega_{0}\right)$ and

$$
\rho:=\alpha^{-1} c^{*}(l-r)<1
$$

then for the solution $u(x)$ of the problem(1.1)-(1.2) the following estimates hold:

$$
\|u\|_{\infty} \leqslant C_{0}, \quad\left|u^{\prime}(x)\right| \leqslant C\left(1+e^{-\frac{\alpha x}{\varepsilon}} / \varepsilon\right), \quad 0 \leqslant x \leqslant l,
$$

where $F(x)=-f(x, 0,0)$,

$$
C_{0}=\left(|\varphi(0)|+|A|+\alpha^{-1}\|F\|_{1}+\alpha^{-1} c^{*}\|\varphi\|_{1,0}\right)(1-\rho)^{-1},
$$

providing that $\frac{\partial f}{\partial x}(x, u, v)$ is bounded for $x \in \bar{\Omega}$ and $|u|,|v| \leqslant C_{0}$.

Proof. We rewrite (1.1) in the form

$$
\varepsilon u^{\prime \prime}+a(x) u^{\prime}-b(x) u+c(x) u(x-r)=F(x),
$$

with

$$
b(x)=\frac{\partial f}{\partial u}(x, \tilde{u}, \tilde{v}), c(x)=\frac{\partial f}{\partial v}(x, \tilde{u}, \tilde{v}),
$$

$\tilde{u}=\gamma u, \tilde{v}=\gamma u(x-r),(0<\gamma<1)$-intermediate values. After using the Maximum Principle for the differential operator $L_{*} u=\varepsilon u^{\prime \prime}+a(x) u^{\prime}-b(x) u$, with first type boundary conditions, we get $|u(x)| \leqslant w(x)$, where $w(x)$ is the solution of the boundary value problem:

$$
\begin{aligned}
& -\varepsilon w^{\prime \prime}-a(x) w^{\prime}=|c(x) u(x-r)|-|F(x)|, \\
& |w(0)|=|\varphi(0)|, \quad|w(l)|=|A| .
\end{aligned}
$$

The further analysis is almost identical to that of [1]. 
Lemma 2. Let $a(x) \in C^{1}(\bar{\Omega}), f(x, \cdot, \cdot) \in C^{1}\left(\bar{\Omega}, \mathbb{R}^{2}\right), \varphi(x) \in C^{1}\left(\Omega_{0}\right)$ and condition (2.1) be satisfied. Then

$$
\left|u^{\prime \prime}(x)\right| \leqslant\left\{\begin{array}{l}
C\left(1+\frac{1}{\varepsilon^{2}} e^{-\frac{\alpha x}{\varepsilon}}\right), x \in \Omega_{1}, \\
C\left(1+\frac{x-r}{\varepsilon^{2}} e^{-\frac{\alpha(x-r)}{\varepsilon}}\right), x \in \Omega_{2},
\end{array}\right.
$$

providing that $\frac{\partial f}{\partial x}(x, u, v)$ is bounded for $x \in \bar{\Omega}$.

Proof. Differentiating (1.1), we get

$$
\varepsilon u^{\prime \prime \prime}(x)+a(x) u^{\prime \prime}(x)=\Phi(x),
$$

with

$$
\begin{aligned}
\Phi(x)= & -\frac{\partial f}{\partial x}(x, u(x), u(x-r))-\left[a^{\prime}(x)+\frac{\partial f}{\partial u}(x, u(x), u(x-r))\right] u^{\prime}(x) \\
& -\frac{\partial f}{\partial v}(x, u(x), u(x-r)) u^{\prime}(x-r) .
\end{aligned}
$$

Hence for $x \in \Omega_{1}$ we have

$$
\begin{aligned}
& u^{\prime \prime}(x)=u^{\prime \prime}(0) e^{-\frac{1}{\varepsilon} \int_{0}^{x} a(\eta) d \eta}+\frac{1}{\varepsilon} \int_{0}^{x} \Phi(\xi) e^{-\frac{1}{\varepsilon} \int_{\xi}^{x} \alpha d \eta} d \xi, \\
& \left|u^{\prime \prime}(x)\right| \leqslant\left|u^{\prime \prime}(0)\right| e^{-\frac{1}{\varepsilon} \int_{0}^{x} \alpha d \eta} \\
& \quad+\frac{1}{\varepsilon} \int_{0}^{x} \mid-\frac{\partial f}{\partial \xi}(\xi, u(\xi), u(\xi-r))-\left[a^{\prime}(\xi)+\frac{\partial f}{\partial u}(\xi, u(\xi), u(\xi-r))\right] u^{\prime}(\xi) \\
& \quad-\frac{\partial f}{\partial v}(\xi, u(\xi), u(\xi-r)) u^{\prime}(\xi-r) \mid e^{-\frac{1}{\varepsilon} \int_{\xi}^{x} \alpha d \eta} d \xi .
\end{aligned}
$$

Moreover, from (1.1) it follows that

$$
\left|u^{\prime \prime}(0)\right| \leqslant \frac{1}{\varepsilon}\left[|f(0, u(0), u(-r))|+|a(0)|\left|u^{\prime}(0)\right|\right] .
$$

This, together with Lemma 1 implies that

$$
\left|u^{\prime \prime}(0)\right| \leqslant C / \varepsilon^{2} .
$$

From the (2.4), (2.5), with the use of (1) we derive

$$
\begin{aligned}
& \left|u^{\prime \prime}(x)\right| \leqslant \frac{C}{\varepsilon^{2}} e^{-\frac{\alpha x}{\varepsilon}}+\frac{1}{\varepsilon} C \int_{0}^{x}\left(1+\left|u^{\prime}(\xi)\right|+\left|u^{\prime}(\xi-r)\right|\right) e^{-\frac{1}{\varepsilon} \int_{\xi}^{x} \alpha d \eta} d \xi \\
& \quad \leqslant \frac{C}{\varepsilon^{2}} e^{-\frac{\alpha x}{\varepsilon}}+\frac{1}{\varepsilon} C \int_{0}^{x}\left(1+\frac{1}{\varepsilon} e^{-\frac{\alpha \xi}{\varepsilon}}\right) e^{-\frac{\alpha(x-\xi)}{\varepsilon}} d \xi+\frac{1}{\varepsilon} C \int_{0}^{x}\left|\varphi^{\prime}(\xi-r)\right| e^{-\frac{\alpha(x-\xi)}{\varepsilon}} d \xi \\
& \leqslant C\left(1+\frac{1}{\varepsilon^{2}} e^{-\frac{\alpha x}{\varepsilon}}+\frac{1}{\varepsilon} \int_{0}^{x}\left(\frac{1}{\varepsilon} e^{-\frac{\alpha \xi}{\varepsilon}}\right) e^{-\frac{\alpha(x-\xi)}{\varepsilon}} d \xi\right), \quad x \in \Omega_{1},
\end{aligned}
$$

which leads to first bound in (2.2). 
Further, for $x \in \Omega_{2}$, from (2.3) we obtain

$$
\begin{aligned}
& u^{\prime \prime}(x)=u^{\prime \prime}(r) e^{-\frac{1}{\varepsilon} \int_{r}^{x} a(\eta) d \eta}+\frac{1}{\varepsilon} \int_{r}^{x} \Phi(\xi) e^{-\frac{1}{\varepsilon} \int_{\xi}^{x} \alpha d \eta} d \xi, \\
& \left|u^{\prime \prime}(x)\right| \leqslant\left|u^{\prime \prime}(r)\right| e^{-\frac{1}{\varepsilon} \int_{r}^{x} \alpha d \eta} \\
& \quad+\frac{1}{\varepsilon} \int_{r}^{x} \mid-\frac{\partial f}{\partial \xi}(\xi, u(\xi), u(\xi-r))-\left[a^{\prime}(\xi)+\frac{\partial f}{\partial u}(\xi, u(\xi), u(\xi-r))\right] u^{\prime}(\xi) \\
& -\frac{\partial f}{\partial v}(\xi, u(\xi), u(\xi-r)) u^{\prime}(\xi-r) \mid e^{-\frac{1}{\varepsilon} \int_{\xi}^{x} \alpha d \eta} d \xi .
\end{aligned}
$$

From this by the first bound in (2.2) we can write

$$
\left|u^{\prime \prime}(r)\right| \leqslant C\left(1+\varepsilon^{-2} e^{-\frac{\alpha r}{\varepsilon}}\right),
$$

so $\left|u^{\prime \prime}(r)\right| \leqslant C$. After inserting this estimate and Lemma 1 in (2.6), we arrive at

$$
\begin{aligned}
\left|u^{\prime \prime}(x)\right| & \leqslant C e^{-\frac{\alpha(x-r)}{\varepsilon}}+\frac{1}{\varepsilon} C \int_{r}^{x}\left(1+\left|u^{\prime}(\xi)\right|+\left|u^{\prime}(\xi-r)\right|\right) e^{-\frac{\alpha(x-\xi)}{\varepsilon}} d \xi \\
& \leqslant C\left(1+\frac{1}{\varepsilon} \int_{r}^{x}\left(\frac{1}{\varepsilon} e^{-\frac{\alpha \xi}{\varepsilon}}+\frac{1}{\varepsilon} e^{-\frac{\alpha(\xi-r)}{\varepsilon}}\right) e^{-\frac{\alpha(x-\xi)}{\varepsilon}} d \xi\right) \\
& =C\left(1+\frac{1}{\varepsilon} \int_{r}^{x}\left(\frac{1}{\varepsilon} e^{-\frac{\alpha(\xi-r)}{\varepsilon}}\right) e^{-\frac{\alpha(x-\xi)}{\varepsilon}} d \xi\right),
\end{aligned}
$$

which implies the second bound in (2.2).

\section{Discrete problem}

In this section, we will develop the new finite difference schemes for (1.1)-(1.2). Let $\omega_{N}$ be a nonuniform mesh on $\Omega$ :

$$
\bar{\omega}_{N}=\left\{0=x_{0}<x_{1}<\ldots<x_{N_{0}}<\ldots<x_{N-1}<x_{N}=l, h_{i}=x_{i}-x_{i-1}\right\} .
$$

For simplicity, we will suppose that $N / 2=N_{0}$ (or $l=2 r$ ) is integer, i.e., $x_{N_{0}}=r$. Before describing our numerical method, we introduce some notation for the mesh functions. For any mesh function $g(x)$ defined on $\bar{\omega}_{N}$ we use

$$
\begin{aligned}
& g_{i}=g\left(x_{i}\right), \quad g_{\bar{x}, i}=\frac{g_{i}-g_{i-1}}{h_{i}}, \quad g_{x, i}=\frac{g_{i+1}-g_{i}}{h_{i+1}}, \quad g_{\bar{x} \hat{x}, i}=\frac{g_{x, i}-g_{\bar{x}, i}}{\hbar_{i}}, \\
& \hbar_{i}=\frac{h_{i}+h_{i+1}}{2},\|g\|_{\infty} \equiv\|g\|_{\infty, \bar{\omega}_{N}}=\max _{0 \leqslant i \leqslant N}\left|g_{i}\right|, \quad\|g\|_{1}=\|g\|_{1, \omega_{N}}=\sum_{i=1}^{N-1} \hbar_{i}\left|g_{i}\right| .
\end{aligned}
$$

The approach of generating difference method is through the integral identity

$$
\hbar_{i}^{-1} \int_{x_{i-1}}^{x_{i+1}} L u(x) \psi_{i}(x) d x=0, \quad 0<i<N
$$


with the linear basis functions

$$
\psi_{i}(x)=\left\{\begin{array}{l}
\frac{x-x_{i-1}}{h_{i}}, x_{i-1}<x<x_{i}, \\
\frac{x_{i+1}-x}{h_{i+1}}, x_{i}<x<x_{i+1}, \\
0, x \notin\left(x_{i-1}, x_{i+1}\right) .
\end{array}\right.
$$

We easily obtain

$$
\varepsilon u_{\bar{x} \hat{x}, i}+a_{i} u_{x, i}+f\left(x_{i}, u_{i}, u_{i-N_{0}}\right)+R_{i}=0, \quad i=1,2, \ldots, N-1,
$$

with remainder term

$$
R_{i}=R_{i}^{(1)}+R_{i}^{(2)}+R_{i}^{(3)}
$$

$$
\begin{aligned}
& R_{i}^{(1)}=a_{i}\left[\hbar_{i}^{-1} \int_{x_{i-1}}^{x_{i+1}} \psi_{i}(x) u^{\prime}(x) d x-u_{x, i}\right], \\
& R_{i}^{(2)}=\hbar_{i}^{-1} \int_{x_{i-1}}^{x_{i+1}}\left[a(x)-a\left(x_{i}\right)\right] u^{\prime}(x) \psi_{i}(x) d x,
\end{aligned}
$$

$R_{i}^{(3)}=\hbar_{i}^{-1} \int_{x_{i-1}}^{x_{i+1}} d x \psi_{i}(x) \int_{x_{i-1}}^{x_{i+1}} \frac{d}{d x} f(\xi, u(\xi), u(\xi-r))\left[T_{0}(x-\xi)-T_{0}\left(x_{i}-\xi\right)\right] d \xi$

$\left(T_{n}(\lambda)=\lambda^{n} / n !, \lambda \geqslant 0 ; T_{n}(\lambda)=0, \lambda<0\right)$.

Based on foregoing, we propose the following difference scheme for approximating (1.1)-(1.2):

$$
\begin{aligned}
& \ell y_{i} \equiv \varepsilon y_{\bar{x} \hat{x}, i}+a_{i} y_{x, i}+f\left(x_{i}, y_{i}, y_{i-N_{0}}\right)=0, \quad 0<i<N \\
& y_{i}=\varphi_{i}, \quad-N_{0} \leqslant i \leqslant 0, \quad y_{N}=B .
\end{aligned}
$$

The difference scheme (3.2)-(3.3), in order to be $\varepsilon$-uniform convergent, we will use the special nonuniform mesh on $\bar{\omega}_{N}$, with Shishkin's transition point. For the even number $N_{0}$, the piecewise uniform mesh $\omega_{N, 1}$ divides each of the interval $[0, \sigma]$ and $[\sigma, r]$ into $N_{0} / 2$ equidistant subintervals, where the transition point $\sigma$, which separates the fine and coarse portions of the mesh is obtained by taking

$$
\sigma=\min \left\{r / 2, \alpha^{-1} \varepsilon \ln N_{0}\right\}
$$

so $\omega_{N}=\omega_{N, 1} \cup \omega_{N, 2}$,

$$
\begin{aligned}
& \omega_{N, 1}=\left\{\begin{array}{l}
x_{i}=i h^{(1)}, i=1,2, \ldots, N_{0} / 2, h^{(1)}=2 \sigma / N_{0} ; \\
x_{i}=\sigma+\left(i-N_{0} / 2\right) h^{(2)}, i=N_{0} / 2+1, \ldots, N_{0}, h^{(2)}=2(r-\sigma) / N_{0}
\end{array}\right\}, \\
& \omega_{N, 2}=\left\{x_{i}=r+\left(i-N_{0}\right) h^{(3)}, i=N_{0}+1, \ldots, N-1, h^{(3)}=r / N_{0}\right\} .
\end{aligned}
$$

In the rest of the paper we only consider this mesh.

\section{Uniform error estimates}

In order to investigate the convergence of present method, note that the error function $z=y-u, x \in \bar{\omega}_{N}$ is the solution of the discrete problem

$$
\begin{aligned}
& \varepsilon z_{\bar{x} \hat{x}, i}+a_{i} z_{x, i}+f\left(x_{i}, y_{i}, y_{i-N_{0}}\right)-f\left(x_{i}, u_{i}, u_{i-N_{0}}\right)=R_{i}, \quad 0<i<N, \\
& z_{i}=0, \quad-N_{0} \leqslant i \leqslant 0 \quad, \quad z_{N}=0,
\end{aligned}
$$

where the truncation error $R_{i}$ is given by (3.1). 
Lemma 3. If $a(x) \in C^{1}(\bar{\Omega}), f(x, \cdot, \cdot) \in C^{1}\left(\bar{\Omega}, \mathbb{R}^{2}\right)$ and $\varphi(x) \in C^{1}\left(\Omega_{0}\right)$, then for the truncation error $R_{i}$ we have

$$
\|R\|_{1} \leqslant C N_{0}^{-1} \ln N_{0} .
$$

Proof. We will estimate $R_{i}^{(k)}(k=1,2,3)$ separately. Let us first proves bound for $R_{i}^{(1)}$. Using interpolating quadrature formula on interval $\left(x_{i-1}, x_{i+1}\right)$ with respect to points $x_{i}, x_{i+1}$ with remainder term in integral form we can write

$$
\begin{aligned}
u(x)= & h_{i+1}^{-1}\left(x_{i+1}-x\right) u_{i}+h_{i+1}^{-1}\left(x-x_{i}\right) u_{i+1}+r(x)=u_{i}+\left(x-x_{i}\right) u_{x, i}+r(x), \\
r(x)= & \int_{x_{i-1}}^{x_{i+1}}\left[T_{1}(x-\xi)-h_{i+1}^{-1}\left(x_{i+1}-x\right) T_{1}\left(x_{i}-\xi\right)\right. \\
& \left.-h_{i+1}^{-1}\left(x_{i+1}-\xi\right)\left(x-x_{i}\right)\right] u^{\prime \prime}(\xi) d \xi
\end{aligned}
$$

and from here it follows that

$$
u^{\prime}(x)=u_{x, i}+\int_{x_{i-1}}^{x_{i+1}}\left[T_{0}(x-\xi)+h_{i+1}^{-1} T_{1}\left(x_{i}-\xi\right)-h_{i+1}^{-1}\left(x_{i+1}-\xi\right)\right] u^{\prime \prime}(\xi) d \xi .
$$

Multiplying it by $\psi_{i}(x)$ and integrating over $\left(x_{i-1}, x_{i+1}\right)$ we obtain

$$
\begin{aligned}
& \hbar_{i}^{-1} \int_{x_{i-1}}^{x_{i+1}} \psi_{i}(x) u^{\prime}(x) d x=u_{x, i}+\hbar_{i}^{-1} \int_{x_{i-1}}^{x_{i+1}} d x \psi_{i}(x) \int_{x_{i-1}}^{x_{i+1}}\left[T_{0}(x-\xi)\right. \\
& \left.\quad+h_{i+1}^{-1} T_{1}\left(x_{i}-\xi\right)-h_{i+1}^{-1}\left(x_{i+1}-\xi\right)\right] u^{\prime \prime}(\xi) d \xi=u_{x, i}+\hbar_{i}^{-1} \int_{x_{i-1}}^{x_{i+1}} \Phi_{i}(\xi) u^{\prime \prime}(\xi) d \xi
\end{aligned}
$$

where

$$
\begin{aligned}
\Phi_{i}(\xi) & =\int_{x_{i-1}}^{x_{i+1}} \psi_{i}(x)\left[T_{0}(x-\xi)+h_{i+1}^{-1} T_{1}\left(x_{i}-\xi\right)-h_{i+1}^{-1}\left(x_{i+1}-\xi\right)\right] d x \\
& =\left\{\begin{array}{l}
-\frac{\left(\xi-x_{i-1}\right)^{2}}{2 h_{i}}, \xi<x_{i} \\
-\frac{\left(x_{i+1}-\xi\right)}{2 h_{i+1}}\left(\xi-x_{i-1}\right), \xi>x_{i} .
\end{array}\right.
\end{aligned}
$$

That is

$$
R_{i}^{(1)}=a_{i} \hbar_{i}^{-1} \int_{x_{i-1}}^{x_{i+1}} \Phi_{i}(\xi) u^{\prime \prime}(\xi) d \xi
$$

We consider first the case $\sigma=r / 2$, and so $\alpha^{-1} \varepsilon \ln N_{0}>r / 2$. Then $h^{(1)}=$ $h^{(2)}=h^{(3)}=r N_{0}^{-1}$ and

$$
\left|R_{i}^{(1)}\right| \leqslant C \int_{x_{i-1}}^{x_{i+1}}\left|u^{\prime \prime}(x)\right| d x, 1 \leqslant i \leqslant N-1 .
$$

Therefore

$$
\begin{aligned}
& \left\|R^{(1)}\right\|_{1} \leqslant C N_{0}^{-1} \int_{0}^{l}\left|u^{\prime \prime}(x)\right| d x \\
& \quad \leqslant C\left(N_{0}^{-1}+N_{0}^{-1} \int_{0}^{r} \frac{1}{\varepsilon^{2}} e^{-\frac{\alpha x}{\varepsilon}} d x+N_{0}^{-1} \int_{r}^{l} \frac{x-r}{\varepsilon^{2}} e^{-\frac{\alpha(x-r)}{\varepsilon}} d x\right)
\end{aligned}
$$


and using the inequality $t e^{-t} \leqslant e^{-\frac{t}{2}}(t>0)$ for $t=(x-r) / \varepsilon$ we get

$$
\begin{aligned}
\left\|R^{(1)}\right\|_{1} & \leqslant C\left(N_{0}^{-1}+N_{0}^{-1} \int_{0}^{r} \frac{1}{\varepsilon^{2}} e^{-\frac{\alpha x}{\varepsilon}} d x+N_{0}^{-1} \int_{r}^{l} \frac{1}{\varepsilon} e^{-\frac{\alpha(x-r)}{2 \varepsilon}} d x\right) \\
& \leqslant C\left(N_{0}^{-1}+N_{0}^{-1} / \varepsilon\right) .
\end{aligned}
$$

Thereby, for $\sigma=r / 2$

$$
\left\|R^{(1)}\right\|_{1} \leqslant C N_{0}^{-1} \ln N_{0} .
$$

We now consider the case $\sigma=\alpha^{-1} \varepsilon \ln N_{0}$. For $1 \leqslant i \leqslant N_{0} / 2-1$ from (4.4) we have

$$
\left|R_{i}^{(1)}\right| \leqslant C \int_{x_{i-1}}^{x_{i+1}}\left|u^{\prime \prime}(x)\right| d x \leqslant C\left(h^{(1)}+\frac{1}{\varepsilon^{2}} \int_{x_{i-1}}^{x_{i+1}} e^{-\frac{\alpha x}{\varepsilon}} d x\right) .
$$

Therefore

$$
\begin{aligned}
& \sum_{i=1}^{N_{0} / 2-1} \hbar_{i}\left|R_{i}^{(1)}\right| \leqslant C\left(h^{(1)}+\frac{h^{(1)}}{\varepsilon^{2}} \int_{x_{0}}^{x_{N_{0} / 2-1}} e^{-\frac{\alpha x}{\varepsilon}} d x\right) \\
& \leqslant C\left(h^{(1)}+\alpha^{-1} \frac{h^{(1)}}{\varepsilon}\left(1-e^{-\frac{\alpha x_{0} / 2-1}{\varepsilon}}\right)\right) \leqslant C\left(h^{(1)}+\frac{h^{(1)}}{\varepsilon}\right)=O\left(N_{0}^{-1} \ln N_{0}\right) .
\end{aligned}
$$

For $N_{0} / 2$, using the representation (4.4) and performing summation by parts, we obtain

$$
\hbar_{N_{0} / 2}\left|R_{N_{0} / 2}^{(1)}\right| \leqslant C\left(N_{0}^{-1}+\frac{1}{\varepsilon^{2}} \int_{x_{N_{0} / 2-1}}^{x_{N_{0} / 2+1}}\left|\Phi_{N_{0} / 2}\right| e^{-\frac{\alpha x}{\varepsilon}} d x\right)
$$

since $\Phi_{N_{0} / 2}=0$ both $x_{N_{0} / 2-1}$ and $x_{N_{0} / 2+1}$ points

$$
\begin{aligned}
\hbar_{N_{0} / 2} & \left|R_{N_{0} / 2}^{(1)}\right| \leqslant C\left(N_{0}^{-1}+\frac{1}{\varepsilon} \int_{x_{N_{0} / 2-1}}^{x_{N_{0} / 2}} \frac{x-x_{N_{0} / 2}}{h_{N_{0} / 2}} e^{-\frac{\alpha x}{\varepsilon}} d x\right. \\
& \left.+\frac{1}{\varepsilon} \int_{x_{N_{0} / 2}}^{x_{N_{0} / 2+1}} \frac{\hbar_{N_{0} / 2}}{h_{N_{0} / 2+1}} e^{-\frac{\alpha x}{\varepsilon}} d x\right) \leqslant C\left(N_{0}^{-1}+\frac{1}{\varepsilon} \int_{x_{N_{0} / 2-1}}^{x_{N_{0} / 2+1}} e^{-\frac{\alpha x}{\varepsilon}} d x\right) \\
\leqslant & C\left(N_{0}^{-1}+\frac{\alpha^{-1} \varepsilon}{\varepsilon}\left(e^{-\frac{\alpha x_{N_{0} / 2-1}}{\varepsilon}}-e^{-\frac{\alpha x_{N_{0} / 2+1}}{\varepsilon}}\right)\right) \\
\leqslant & C\left(N_{0}^{-1}+\alpha^{-1} e^{-\frac{\alpha x_{0} / 2-1}{\varepsilon}}\right),
\end{aligned}
$$

from which, clearly

$$
\hbar_{N_{0} / 2}\left|R_{N_{0} / 2}^{(1)}\right| \leqslant C N_{0}^{-1} .
$$

For $N_{0} / 2+1 \leqslant i \leqslant N_{0}-1$ we have

$$
\begin{aligned}
\sum_{i=N_{0} / 2+1}^{N_{0}-1} \hbar_{i}\left|R_{i}^{(1)}\right| & \leqslant C\left(h^{(2)}+\frac{h^{(2)}}{\varepsilon^{2}} \int_{x_{N_{0} / 2+1}}^{x_{N_{0}-1}} e^{-\frac{\alpha x}{\varepsilon}} d x\right) \\
& \leqslant C\left(h^{(2)}+\frac{h^{(2)}}{\varepsilon} e^{-\frac{\alpha x}{N_{0} / 2+h^{(2)}}}\right) \leqslant C\left(h^{(2)}+N_{0}^{-1} \frac{h^{(2)}}{\varepsilon} e^{-\frac{h^{(2)}}{\varepsilon}}\right) .
\end{aligned}
$$


Using the inequality $t e^{-t} \leqslant e^{-t / 2}(t>0)$ for $t=h^{(2)} / \varepsilon$ and taking into account that $h^{(2)} \leqslant 2 r N_{0}^{-1}$, we find that

$$
\sum_{i=N_{0} / 2+1}^{N-1} \hbar_{i}\left|R_{i}^{(1)}\right| \leqslant C N_{0}^{-1} .
$$

For $N_{0} \leqslant i \leqslant N-1$, evidently

$$
\sum_{i=N_{0}}^{N-1} \hbar_{i}\left|R_{i}^{(1)}\right| \leqslant C N_{0}^{-1} .
$$

Consequently, (4.5)-(4.6) imply the desired estimate

$$
\left\|R^{(1)}\right\|_{1} \leqslant C N_{0}^{-1} \ln N_{0},
$$

which will be held true for any $\varepsilon>0$.

Next, for any $\varepsilon>0$ and $a \in C^{1}(\bar{\Omega})$ we have

$$
\begin{aligned}
\left\|R^{(2)}\right\|_{1} & =\sum_{i=1}^{N-1} \hbar_{i}\left|R_{i}^{(2)}\right| \leqslant C N^{-1} \sum_{i=1}^{N} \int_{x_{i-1}}^{x_{i+1}}\left|u^{\prime}(x)\right| d x=C N^{-1} \int_{0}^{l}\left|u^{\prime}(x)\right| d x \\
& \leqslant C N^{-1} \int_{0}^{l} \frac{1}{\varepsilon} e^{-\frac{\alpha x}{\varepsilon}} d x=C N^{-1} \alpha^{-1}\left(1-e^{-\frac{\alpha l}{\varepsilon}}\right)=O\left(N^{-1}\right) .
\end{aligned}
$$

Also, under $f \in C^{1}\left(\bar{\Omega}, \mathbb{R}^{2}\right)$ and from $R_{i}^{(3)}$ taking into account that $\hbar_{i}^{-1} \int_{x_{i-1}}^{x_{i+1}} \psi_{i}(x) d x=1$ we get

$$
\begin{aligned}
\left|R_{i}^{(3)}\right| & \leq C\left(\int_{x_{i-1}}^{x_{i+1}}\left|\frac{\partial f(\xi, u(\xi), u(\xi-r))}{\partial \xi}\right| d \xi+\int_{x_{i-1}}^{x_{i+1}}\left|\frac{\partial f}{\partial u} \frac{d u(\xi)}{d \xi}+\frac{\partial f}{\partial v} \frac{d u(\xi-r)}{d \xi}\right| d \xi\right) \\
& \leq C\left[\hbar_{i}+\int_{x_{i-1}}^{x_{i+1}}\left(\left|u^{\prime}(\xi)\right|+\left|u^{\prime}(\xi-r)\right|\right) d \xi\right] .
\end{aligned}
$$

Hence,

$$
\begin{aligned}
\left\|R^{(3)}\right\|_{1} & =\sum_{i=1}^{N-1} \hbar_{i}\left|R_{i}^{(3)}\right| \leqslant C \sum_{i=1}^{N-1}\left[\hbar_{i}^{2}+\hbar_{i} \int_{x_{i-1}}^{x_{i+1}}\left(\left|u^{\prime}(x)\right|+\left|u^{\prime}(x-r)\right|\right) d x\right] \\
& \leqslant C N^{-1}\left(1+\int_{0}^{l}\left|u^{\prime}(x)\right| d x+\int_{0}^{l}\left|u^{\prime}(x-r)\right| d x\right) \\
& =C N^{-1}\left(2+\int_{-r}^{0}\left|\varphi^{\prime}(s)\right| d s+\int_{0}^{l-r}\left|u^{\prime}(s)\right| d s\right) \\
& \leqslant C N^{-1}\left(2+\|\varphi\|_{1,0}+\alpha^{-1}\left(1-e^{-\frac{\alpha(l-r)}{\varepsilon}}\right)\right)=O\left(N^{-1}\right) .
\end{aligned}
$$

Substituting the estimates (4.7)-(4.8) into (3.1), we arrive at (4.3). 
Lemma 4. Let $z_{i}$ be the solution (4.1)-(4.2) and (2.1) holds true. Then

$$
\|z\|_{\infty, \omega} \leqslant\|R\|_{1, \omega} .
$$

Proof. (4.1) can be rewritten as

$$
\varepsilon z_{\bar{x} \hat{x}, i}+a_{i} z_{x, i}-\widetilde{b}_{i} z_{i}+\widetilde{c}_{i} z_{i-N_{0}}=R_{i}, 0<i<N,
$$

where

$$
\widetilde{b}_{i}=\frac{\partial f}{\partial u}\left(x_{i}, \widetilde{y}_{i}, \widetilde{y}_{i-N_{0}}\right), \widetilde{c}_{i}=\frac{\partial f}{\partial v}\left(x_{i}, \widetilde{y}_{i}, \widetilde{y}_{i-N_{0}}\right),
$$

$\widetilde{y}_{i}, \widetilde{y}_{i-N_{0}}$ intermediate points called for by the mean value theorem.

Here we use the discrete Green's function $G^{h}\left(x_{i}, \xi_{j}\right)$ for the operator

$$
\begin{aligned}
& L^{h} z_{i}:=-\varepsilon z_{\bar{x} \hat{x}, i}-a_{i} z_{x, i}, \quad 1 \leqslant i \leqslant N-1, \\
& z_{0}=z_{N}=0 .
\end{aligned}
$$

As a function of $x_{i}$ for fixed $\xi_{j}$ this function is being defined as

$$
\begin{aligned}
& L^{h} G^{h}\left(x_{i}, \xi_{j}\right)=\delta^{h}\left(x_{i}, \xi_{j}\right), \quad x_{i} \in \omega, \quad \xi_{j} \in \omega, \\
& G^{h}\left(0, \xi_{j}\right)=G^{h}\left(l, \xi_{j}\right), \quad \xi_{j} \in \omega,
\end{aligned}
$$

where $\delta^{h}\left(x_{i}, \xi_{j}\right)=\hbar_{i}^{-1} \delta_{i j}$ and $\delta_{i j}$ is the Kronecker delta. Using Green's function, we can write down the following relation for solution of problem (4.1)-(4.2)

$$
z_{i}=\sum_{j=1}^{N-1} \hbar_{i} G^{h}\left(x_{i}, \xi_{j}\right)\left(\widetilde{c}_{i} z_{j-N_{0}}-R_{j}\right), \quad x_{i} \in \omega .
$$

In the analogous manner as in $[1,2]$ one can show that $0 \leqslant G^{h}\left(x_{i}, \xi_{j}\right) \leqslant \alpha^{-1}$. Then from (4.10) it follows that

$$
\|z\|_{\infty, \omega} \leqslant \alpha^{-1}\left\{c^{*} \sum_{j=1}^{N-1} \hbar_{i}\left|z_{j-N_{0}}\right|+\|R\|_{1, \omega}\right\}
$$

and after replacing $j-N_{0}=k$, we have

$$
\begin{aligned}
& \|z\|_{\infty, \omega} \leqslant \alpha^{-1} c^{*} \sum_{k=1-N_{0}}^{N-N_{0}-1} \hbar_{i}\left|z_{k}\right|+\alpha^{-1}\|R\|_{1, \omega}=\alpha^{-1} c^{*} \sum_{k=1}^{N-N_{0}-1} \hbar_{i}\left|z_{k}\right| \\
& \quad+\alpha^{-1}\|R\|_{1, \omega} \leqslant \alpha^{-1} c^{*}\left(N-N_{0}-1\right) \hbar_{i}\|z\|_{\infty, \omega}+\alpha^{-1}\|R\|_{1, \omega} \\
& \leqslant \rho\|z\|_{\infty, \omega}+\alpha^{-1}\|R\|_{1, \omega},
\end{aligned}
$$

which implies validity of (4.9).

Combining the previous lemmas yield the main result of the paper.

Theorem 1. Let $u$ be the solution of (1.1)-(1.2) and $y$ - the solution of (3.2)(3.3). Then under hypotheses Lemmas 3 and 4

$$
\|y-u\|_{\infty, \bar{\omega}_{N}} \leqslant C N_{0}^{-1} \ln N_{0} .
$$




\section{$5 \quad$ Algorithm and numerical results}

In this section, we shall propose the following iterative technique for solving problem (3.2)-(3.3). In addition, we demonstrate the effectiveness of the present method by applying it to an example of problem (1.1)-(1.2).

$$
\begin{aligned}
& \varepsilon y_{\bar{x} \hat{x}, i}^{(n)}+a_{i} y_{x, i}^{(n)}+f\left(x_{i}, y_{i}^{(n-1)}, y_{i-N_{0}}^{(n-1)}\right) \\
& +\frac{\partial f}{\partial y}\left(x_{i}, y_{i}^{(n-1)}, y_{i-N_{0}}^{(n-1)}\right)\left[y_{i}^{(n)}-y_{i}^{(n-1)}\right]=0,0<i<N, \\
& y_{i}^{(n)}=\varphi_{i}, \quad-N_{0} \leqslant i \leqslant 0, y_{N}^{(n)}=A,
\end{aligned}
$$

$n=1,2, \ldots ; y_{i}^{(0)}$ given $0<i<N$.

Now, we consider the test problem:

$$
\varepsilon u^{\prime \prime}(x)+32\left(x^{2}+9\right) u^{\prime}(x)=3 x^{2}+e^{-u(x)}+\tanh [x+u(x-1)], 0<x<2,
$$

subject to the interval and boundary conditions,

$$
u(x)=x^{2}, \quad-1 \leqslant x \leqslant 0 ; \quad u(2)=1 .
$$

The initial guess in the iteration process is $y_{i}^{(0)}=\left(1-x_{i}\right)^{2}$ and the stopping criterion is

$$
\max _{i}\left|y_{i}^{(n)}-y_{i}^{(n-1)}\right| \leqslant 10^{-5} \text {. }
$$

The exact solution of our test problem is unknown. Therefore we use the double mesh principle to estimate the errors and compute the experimental rates of convergence in our computed solutions. That is, we compare the computed solutions with the solutions on a mesh that is twice as fine (see $[3,5,8,11])$. The error estimates obtained in this way are denoted by:

$$
e_{\varepsilon}^{N}=\max _{i}\left|y_{i}^{\varepsilon, N}-y_{2 i}^{\varepsilon, 2 N}\right|,
$$

the convergence rates are $p_{\varepsilon}^{N}=\log _{2}\left(e_{\varepsilon}^{N} / e_{\varepsilon}^{2 N}\right)$. Approximations to the $\varepsilon$ uniform rates of convergence are estimated by $e^{N}=\max _{\varepsilon} e_{\varepsilon}^{N}$ and the corresponding $\varepsilon$-uniform convergence rates are computed using the formula

$$
p^{N}=\log _{2}\left(e^{N} / e^{2 N}\right) .
$$

The values of $\varepsilon$ for which we solve the test problem are $\varepsilon=2^{-i}, i=4,8, \ldots, 24$. The resulting errors $e^{N}$ and the corresponding numbers $p^{N}$ which are obtained after only a few iterations are listed in Table 1.

\section{Conclusions}

In this paper, we have developed a finite difference method for solving the singularly perturbed boundary-value problem for a nonlinear second order delay differential equation. To solve this problem, a difference scheme on a piecewise uniform mesh was presented. Almost first order convergence, in the discrete 
Table 1. Computed errors and convergence rates of the test problem

\begin{tabular}{llllll}
\hline$\varepsilon$ & $N_{0}=128$ & $N_{0}=256$ & $N_{0}=512$ & $N_{0}=1024$ & $N_{0}=2048$ \\
\hline $2^{-4}$ & 0.0068089 & 0.0038818 & 0.0021810 & 0.0012010 & 0.0006379 \\
& 0.81 & 0.83 & 0.86 & 0.91 & \\
$2^{-8}$ & 0.0068366 & 0.0038578 & 0.0021378 & 0.0011604 & 0.0006123 \\
& 0.83 & 0.85 & 0.88 & 0.92 & \\
$2^{-12}$ & 0.0068922 & 0.0038860 & 0.0021504 & 0.0011642 & 0.0006115 \\
& 0.83 & 0.85 & 0.89 & 0.93 & \\
$2^{-16}$ & 0.0068917 & 0.0038855 & 0.0021499 & 0.0011637 & 0.0006110 \\
& 0.83 & 0.85 & 0.89 & 0.93 & \\
$2^{-20}$ & 0.0068917 & 0.0038855 & 0.0021499 & 0.0011637 & 0.0006110 \\
& 0.83 & 0.85 & 0.89 & 0.93 & \\
$2^{-24}$ & 0.0068917 & 0.0038855 & 0.0021499 & 0.0011637 & 0.0006110 \\
& 0.83 & 0.85 & 0.89 & 0.93 & \\
$e^{N}$ & 0.0068922 & 0.0038860 & 0.0021810 & 0.0012010 & 0.0006379 \\
$p^{N}$ & 0.83 & 0.83 & 0.86 & 0.91 & \\
\hline
\end{tabular}

maximum norm, independently of the perturbation parameter was obtained. Furthermore, the approximate errors and the rates of convergence for test problem was computed for different values of $\varepsilon$ and $N_{0}$ in Table 1 . Numerical results were carried out to show the efficiency and accuracy of the method. Theoretical results represent undergoing more complicated delay differential problems.

\section{References}

[1] G.M. Amiraliyev and E. Cimen. Numerical method for a singularly perturbed convection-diffusion problem with delay. Applied Mathematics and Computation, 216(8):2351-2359, 2010. https://doi.org/10.1016/j.amc.2010.03.080.

[2] V.B. Andreev. The green function and a priori estimates of solutions of monotone three-point singularly perturbed finite-difference schemes. Differential Equations, 37(7):923-933, 2001. https://doi.org/10.1023/A:1011949419389.

[3] M. Cakir. A numerical study on the difference solution of singularly perturbed semilinear problem with integral boundary condition. Mathematical Modelling and Analysis, 21(5):644-658, 2016. https://doi.org/10.3846/13926292.2016.1201702.

[4] E. Cimen. A priori estimates for solution of singularly perturbed boundary value problem with delay in convection term. Journal of Mathematical Analysis, 8(1):202-211, 2017.

[5] E. Cimen and G.M. Amiraliyev. A uniform convergent method for singularly perturbed nonlinear differential-difference equation. Journal of Informatics and Mathematical Sciences, 9(1):191-199, 2017.

[6] E. Cimen and M. Cakir. Numerical treatment of nonlocal boundary value problem with layer behaviour. Bulletin of the Belgian Mathematical Society - Simon Stevin, 24(3):339-352, 2017.

[7] M.W. Derstein, H.M. Gibbs, F.A. Hopf and D.L. Kaplan. Bifurcation gap in a hybrid optically bistable system. Physical Review A, 26(6):3720-3722, 1982. https://doi.org/10.1103/PhysRevA.26.3720. 
[8] E.R. Doolan, J.J.H. Miller and W.H.A. Schilders. Uniform Numerical Methods for Problems with Initial and Boundary Layers. Boole Press, Dublin, 1980.

[9] L.É. Èls'gol'c. Qualitative Methods in Mathematical Analysis, in: Translations of Mathematical Monographs, vol.12. AMS, Providence, RI, 1964.

[10] T. Erneux. Applied Delay Differential Equations. Surveys and Tutorials in the Applied Mathematical Sciences. Springer, New York, 2009. https://doi.org/10.1007/978-0-387-74372-1.

[11] P. Farrell, A. Hegarty, J.H. Miller, E. O'Riordan and G.I. Shishkin. Robust Computational Techniques for Boundary Layers. Applied Mathematics. Chapman Hall/CRC, New York, 2000.

[12] F.Z. Geng and S.P. Qian. Modified reproducing kernel method for singularly perturbed boundary value problems with a delay. Applied Mathematical Modelling, 39(18):5592-5597, 2015. https://doi.org/10.1016/j.apm.2015.01.021.

[13] V.Y. Glizer. Controllability conditions of linear singularly perturbed systems with small state and input delays. Mathematics of Control, Signals, and Systems, 28(1):1-29, 2015. https://doi.org/10.1007/s00498-015-0152-3.

[14] M.K. Kadalbajoo and V.P. Ramesh. Numerical methods on Shishkin mesh for singularly perturbed delay differential equations with a grid adaptation strategy. Applied Mathematics and Computation, 188(2):1816-1831, 2007. https://doi.org/10.1016/j.amc.2006.11.046.

[15] A. Keane, B. Krauskopf and C.M. Postlethwaite. Climate models with delay differential equations. Chaos: An Interdisciplinary Journal of Nonlinear Science, 27(11):1-15, 2017. https://doi.org/10.1063/1.5006923.

[16] C.G. Lange and R.M. Miura. Singular perturbation analysis of boundary value problems for differential-difference equations. SIAM Journal on Applied Mathematics, 42(3):502-531, 1982. https://doi.org/10.1137/0142036.

[17] C.G. Lange and R.M. Miura. Singular perturbation analysis of boundary value problems for differential-difference equations. IV. A nonlinear example with layer behavior. Studies in Applied Mathematics, 84(3):231-273, 1991. https://doi.org/10.1002/sapm1991843231.

[18] L.-B. Liu and Y. Chen. Maximum norm a posteriori error estimates for a singularly perturbed differential difference equation with small delay. Applied Mathematics and Computation, 227(15):801-810, 2014. https://doi.org/10.1016/j.amc.2013.10.085.

[19] A. Longtin and J. Milton. Complex oscillations in the human pupil light reflex with mixed and delayed feedback. Mathematical Biosciences, 90(1-2):183-199, 1988. https://doi.org/10.1016/0025-5564(88)90064-8.

[20] M.C. Mackey and L. Glass. Oscillation and chaos in physiological control systems. Science, $\mathbf{1 9 7}(4300): 287-289, \quad 1977$. https://doi.org/10.1126/science.267326.

[21] J.J.H. Miller, E. O'Riordan and G.I. Shishkin. Fitted Numerical Methods for Singular Perturbation Problems. Error Estimates in the Maximum Norm for Linear Problems in One and Two Dimensions. Revised Edition. World Scientific, Singapore, 2012.

[22] H.G. Roos, M. Stynes and L. Tobiska. Robust Numerical Methods for Singularly Perturbed Differential Equations, 2nd Edt. Springer Series in Computational Mathematics. Springer-Verlag, Berlin, 2008. https://doi.org/10.1007/978-3-54034467-4. 
[23] P.A. Selvi and N. Ramanujam. An iterative numerical method for singularly perturbed reaction-diffusion equations with negative shift. Journal of Computational and Applied Mathematics, 296:10-23, 2016. https://doi.org/10.1016/j.cam.2015.09.003.

[24] R.B. Stein. Some models of neuronal variability. Biophysical Journal, 7(1):37-68, 1967. https://doi.org/10.1016/S0006-3495(67)86574-3.

[25] V. Subburayan and N. Ramanujam. Asymptotic initial value technique for singularly perturbed convection-diffusion delay problems with boundary and weak interior layers. Applied Mathematics Letters, 25(12):2272-2278, 2012. https://doi.org/10.1016/j.aml.2012.06.016.

[26] H. Zarin. On discontinuous Galerkin finite element method for singularly perturbed delay differential equations. Applied Mathematics Letters, 38(1):27-32, 2014. https://doi.org/10.1016/j.aml.2014.06.013. 